\title{
Is PSR B0943+10 a low-mass quark star?
}

\author{
Y. L. Yue ${ }^{1}$, X. H. Cui ${ }^{1}$, and R. X. Xu ${ }^{2,1}$ \\ ${ }^{1}$ School of Physics, Peking University, Beijing 100871, China \\ ${ }^{2}$ CCAST (World Laboratory), P.O. Box 8730, Beijing 100080, China
}

\begin{abstract}
A recent X-ray observation has shown that the radio pulsar PSR B0943+10, with clear drifting subpulses, has a much smaller polar cap area than that of conventional pulsars with mass of $\sim M_{\odot}$ and radius of $\sim 10 \mathrm{~km}$. Zhang et al. (2005) addressed then that this new result conflicts with the standard vacuum gap model. Nonetheless, the discrepancy could be explained if PSR B0943+10 is actually a low-mass quark star. It is found that the potential drop in the open-field-line region of oblique pulsars (i.e., inclination angle $\alpha \neq 0$ ) might be $\sim 10^{2}$ times that of aligned pulsars, and that PSR B0943+10 with $\alpha=12.4^{\circ}$ could be well above the deathline. We thus conclude that the Ruderman-Sutherland-type vacuum gap model still works well for this pulsar if it is a bare quark star with a mass of $\sim 0.02 M_{\odot}$ and a radius of $\sim 2.6 \mathrm{~km}$.
\end{abstract}

Subject headings: dense matter — pulsars: general — pulsars: individual (PSR B0943+10) — stars: neutron

\section{Introduction}

The nature and emission mechanism of pulsars remain as puzzles for nearly forty years. There are various kinds of emission models for the particle acceleration in pulsar magnetosphere, such as the vacuum gap (e.g., Ruderman \& Sutherland 1975, hereafter RS75), the space-charge-limited flow (e.g., Arons \& Scharlemenn 1979), the outer gap (e.g., Cheng et al. 1986), and the core and annular gaps (e.g., Qiao et al. 2004). Due to the limits of observations and the difficulties in the electrodynamics of pulsar magnetosphere, we still could not know which one really works. An interesting phenomena to discriminate these models is pulsar subpulse drifting. RS75 vacuum gap model is by far the most successful model to understand this drifting subpulse phenomenon. The model introduces polar cap sparks, which demonstrate $\mathbf{E} \times \mathbf{B}$ drift around the magnetic axis. The pulsar PSR B0943+10 is one of the best-studied subpulse-drifting pulsars. With spin period $P=1.1 \mathrm{~s}$ and period derivative $\dot{P}=3.5 \times 10^{-15} \mathrm{~s} / \mathrm{s}$, PSR B0943+10 is not special on the $P$ $\dot{P}$ diagram. The distance of this source is $0.63 \pm 0.10 \mathrm{kpc}$, which is derived from its dispersion measure (Zhang et al. 2005). By fitting its polarization data, Lyne \& Manchester (1988) obtained an inclination angle (angle between the spin axis and the magnetic axis) of $\alpha=12.4^{\circ}$ and a view angle (angle between the spin axis and the line-of-sight) of $\zeta=18.2^{\circ}$. Deshpande \& Rankin (1999, 2001) observed the pulsar and identified 20 sparks rotating with a period of $P_{3}=37 P$. 
In the RS75 vacuum gap model, about half of the energetic particles would hit the polar gap surface, so the polar gap will be heated to emit X-rays. Thermal X-ray emission from the cap region should then be observable. In order to test the vacuum gap model, Zhang et al. (2005) observed PSR B0943+10 with XMM-Newton, but obtained a rather small thermal polar cap area, $A=10^{3}[T /(3 \mathrm{MK})]^{-4} \mathrm{~m}^{2}$, where $T$ is the polar cap surface temperature (with $1-\sigma$ error, $A=$ $0.3 \times 10^{3} \mathrm{~m}^{2} \sim 5 \times 10^{3} \mathrm{~m}^{2}$, and $\left.T=2.0 \mathrm{MK} \sim 4.2 \mathrm{MK}\right)$, whereas a power-law fit is also acceptable (a detail discussion about this issue will be presented in $§ 3$ ). Though the distance of PSR B0943+10 $(0.63 \pm 0.10 \mathrm{kpc})$ (Zhang et al. 2005) is not very accurate, its uncertainty could not be significant comparing to that of polar cap area. The conventional RS75 polar cap area, $6 \times 10^{4} \mathrm{~m}^{2}$, would be then ten times more that observed. Therefore, the observational uncertainties might not be able to explain this discrepancy. To alleviate this difficulty, Zhang et al. (2005) suggested a strong multipole magnetic field in the polar cap region (see also Gil et al. 2005, 2006) for this pulsar.

Though RS75 vacuum gap model with a user-friendly nature is successful to explain subpulse drifting, there are unfortunately two drawbacks. One is the so-called binding energy problem. RS75 model requires that the binding energy of the ions on the neutron star surface should be larger than $\sim 10 \mathrm{keV}$, which should be in doubt (e.g., Xu et al. 1999). The other is: RS75 model could not be able to apply to half of radio pulsars, the antipulsars with $\boldsymbol{\Omega} \cdot \mathbf{B}>0$. The RS75 model may works for $\boldsymbol{\Omega} \cdot \mathbf{B}<0$ if ions can be bound on the surface, but in the case of $\boldsymbol{\Omega} \cdot \mathbf{B}>0$, negatively charged electrons are required to be bound on the surface, which can hardly be attained. A hypothetical plasma-phase-condensation (i.e., magnetic metal) transition was suggested (Lai \& Salpeter 1997) for neutron star atmospheres with high field $B$ and low temperature $T$, and was applied to interpret the binding of electrons (Usov \& Melrose 1995) and the thermal X-ray spectrum (Turolla et al. 2004). Some calculations show that magnetic metal may form if $B \gtrsim 10^{13} \mathrm{G}$ and surface temperature $T<3.7 \times 10^{5} \mathrm{~K}$ (Usov \& Melrose 1995). Actually, few pulsars can fulfill these two criteria simultaneously, so the problem for antipulsars still exists. In order to overcome these two difficulties, some new ideas are proposed, e.g., non-dipolar surface magnetic fields (Gil et al. 2002), a partial flow of iron ions (Gil et al. 2003), and quark stars without crusts (Xu et al. 1999).

Quark stars are composed of unconfined free quarks and gluons, which were proposed soon after Gell-Mann's idea of quarks and were studied extensively ever since (e.g., Ivanenko \& Kurdgelaidze 1969; Itoh 1970; Bodmer 1971; Witten 1984; Alcock et al. 1986, see Xu (2003a) for a short review). Though there are some observational hints that pulsars may be quark stars, whether pulsars are really normal neutron stars or quark stars is still an open question. It was generally believed that only strange stars with crusts (mass $\sim 10^{-5} M_{\odot}$ ), being similar to the outer parts of neutron stars, could work as radio pulsars, because a bare quark surface can not supply particles to form pulsar's magnetospheres and a bare strange star may readily accrete matter from its "dirty" environment (Alcock et al. 1986). However, this view was re-considered by Xu \& Qiao (1998), who addressed that bare strange stars can also have magnetospheres and thus radiate radio waves, since the vacuum in strong electromagnetic static fields just above the quark surfaces is unstable and would create 
$e^{ \pm}$pairs ${ }^{1}$ although no charged particles can be pulled out from the surfaces. Particularly, Xu et al. (1999) have argued then that PSR B0943+10 is a bare strange quark star rather than a normal neutron star, which solves the binding energy problem as well as the antipulsar problem in a very simple way. It was also found that, due to rapid rotation and strong magnetic field, a crust could hardly form even in an accretion phase of a bare strange star unless the accretion rate is much higher than the Eddington rate (Xu et al. 2001; Xu 2002).

In this letter, we investigated PSR B0943+10 with the new observation by Zhang et al. (2005) under this quark star model. In the model, the polar cap area problem would be solved naturally because a quark star could have a low mass, a small radius (Alcock et al. 1986), and consequently a small polar cap area. The binding energy problem would not be faced under the bare quark star model since the binding energy of quarks and electrons on the quark surfaces should be high enough for RS75-type vacuum gaps to work in both cases of $\boldsymbol{\Omega} \cdot \mathbf{B}>0$ and $\boldsymbol{\Omega} \cdot \mathbf{B}<0$ (Xu et al. 1999, 2001).

\section{The Model}

For an aligned pulsar, as assumed in RS75, the polar cap radius is $r_{\mathrm{pc}}=\left(2 \pi R^{3} / c P\right)^{1 / 2}$, where $R$ is the star radius and $c$ is the light speed. The polar cap area of PSR B0943+10 is $A_{\mathrm{pc}}=\pi r_{\mathrm{pc}}^{2} \simeq 6 \times 10^{4} \mathrm{~m}^{2}$ when using $R=10 \mathrm{~km}$ and $P=1.1 \mathrm{~s}$. This is much larger than the observational result of $\sim 10^{3} \mathrm{~m}^{2}$ (Zhang et al. 2005). If PSR B0943+10 is a quark star, the above problem would be solved because a quark star could have a low mass and a small radius (Alcock et al. 1986). Applying $A_{\mathrm{pc}} \simeq 10^{3} \mathrm{~m}^{2}$, one obtains that the star radius to fit the observation is only $2.6 \mathrm{~km}$. The internal density of a low-mass quark star is almost homogeneous in this case. The star's mass can be well approximated by $M \simeq(4 / 3) \pi R^{3} \rho$, where $\rho$ is the density of the quark star. The density $\rho$ could be a few times of the saturation nuclear density $\rho_{\mathrm{n}}$. The exact value of $\rho$ is not clear since no strong constrain has been obtained from experiments or observations. Using a typical density $\rho=2 \rho_{\mathrm{n}}$, we can get the star's mass $M \simeq 0.019 M_{\odot}$. It is actually unclear what the phasetransition density is in the regime of high-density but low-temperature, although lattice quantum chromodynamics (QCD) shows the critical temperature is $(150 \sim 200) \mathrm{MeV}$ for temperaturedominated case. Nevertheless, the critical density could be only about $2 \rho_{\mathrm{n}}$ if neutrons (and protons) keep about $1 \mathrm{fm}$ in radius. The average density of low-mass strange quark stars could be about $4 \bar{B} \sim(4.4 \sim 8.0) \times 10^{14} \mathrm{~g} \mathrm{~cm}^{-3}$, where the bag constant $\bar{B}$ is reasonably $(60 \sim 110) \mathrm{MeV} \mathrm{fm}{ }^{3}$. Even for an extreme case of $\bar{B}=250 \mathrm{MeV} \mathrm{fm}{ }^{3}$, we have $\rho=1.8 \times 10^{15} \mathrm{~g} \mathrm{~cm}^{-3}\left(\sim 6.7 \rho_{\mathrm{n}}\right), M=0.06 M_{\odot}$, and the redshift factor $R_{\infty} / R=\left(1-R_{\mathrm{g}} / R\right)^{1 / 2}=1.04$, where $R_{\mathrm{g}}=2 G M / c^{2}$. This shows that the general relativistic effect correction is very small and can be omitted for low mass quark stars.

An effective electric force to power a pulsar in RS75 model results from a potential drop between

\footnotetext{
${ }^{1}$ This is the condition that the RS75-type vacuum gap model requires.
} 
the magnetic axis (also the spin axis for aligned pulsars) and the last open field line. The potential drop is $\Phi=\Omega B R^{2} \sin ^{2} \theta /(2 c)$, where $\Omega=2 \pi / P, B$ is the star surface magnetic field strength at the magnetic polar, $\sin \theta=r_{\mathrm{pc}} / R=\left(R / R_{\mathrm{LC}}\right)^{1 / 2}, R_{\mathrm{LC}}=c / \Omega$ is the light cylinder radius, and $\theta$ is half the opening angle of the polar cap. We have effectively, $\Phi \simeq 3 \times 10^{16} R_{6}^{2} B_{12} P \sin ^{2} \theta / P$ Volts, where $R_{6}=R /\left(10^{6} \mathrm{~cm}\right), B_{12}=B /\left(10^{12} \mathrm{G}\right)$, and $P$ in units of $1 \mathrm{~s}$. One can get $\Phi=6.6 \times 10^{11} \mathrm{~V}<\Phi_{\mathrm{c}} \sim$ $10^{12} \mathrm{~V}$ for PSR B0943+10 if it has $M=0.019 M_{\odot}$ and $R=2.6 \mathrm{~km}$, where $\Phi_{\mathrm{c}}$ is the critical voltage which is obtained by requiring all the observed radio pulsars have $\Phi>\Phi_{\mathrm{c}}$. The field $B=6.8 \times 10^{12}$ $\mathrm{G}$ is obtained from Eq.(1). If $\Phi<\Phi_{\mathrm{c}}$, particles would not be accelerated to have enough energy to form sparks, and thus the star should not give out radio emission. Actually, the death-line criteria is not very certain yet, so we use a generally accepted constant potential drop of $\Phi_{\mathrm{c}} \sim 10^{12} \mathrm{~V}$ for the sake of simplicity. Since the criteria is not strict, a quark star with a potential drop of $6.6 \times 10^{11} \mathrm{~V}$ might be able to give out radio emission.

Nevertheless, the following calculation shows that the pulsar's potential drop could also be much larger than $\Phi_{\mathrm{c}}$ if the inclination angle $\alpha=12.4^{\circ}$ is considered, since the assumption of alignment in RS75 is a rather strong assumption. For the general case of oblique rotators $(\alpha \neq$ $0), \mathrm{Xu} \&$ Qiao (2001) proposed that the magnetic momentum of dipole magnetic field could be expressed as $\vec{\mu}=\vec{\mu}_{\perp}+\vec{\mu}_{\|}$, where $\mu_{\perp}=\mu \sin \alpha$ and $\mu_{\|}=\mu \cos \alpha$. In this way, the energy-lose rate $\dot{E}$ consists two parts: that of magnetic dipole radiation $\left(\dot{E}_{\perp}\right)$ and that of particle ejection due to the unipolar generator $\left(\dot{E}_{\|}\right)$. Xu \& Qiao (2001) showed that the sum of these two parts are of the same order of pure dipole radiation. Therefore, the canonical relation $B \propto(P \dot{P})^{1 / 2}$ can be approximately valid. We use the form, with a correction for $M$ and $R$, to estimate the magnetic field,

$$
B \simeq 6.4 \times 10^{19}(P \dot{P})^{1 / 2}\left(\frac{M}{1.4 M_{\odot}}\right)^{1 / 2}\left(\frac{R}{10 \mathrm{~km}}\right)^{-2} \quad \mathrm{G} .
$$

Consequently, the maximum potential drop would be

$$
\Phi_{\max } \simeq \frac{\Omega B R^{2}}{2 c} \cos \alpha\left(\sin ^{2} \theta_{2}-\sin ^{2} \theta_{1}\right)
$$

where $\theta_{1}=\alpha-\theta$ (if $\alpha-\theta>0$ ) or 0 (if $\alpha-\theta \leq 0$ ) and $\theta_{2}=\alpha+\theta$ (if $\alpha+\theta<90^{\circ}$ ) or $90^{\circ}$ (if $\left.\alpha+\theta \geq 90^{\circ}\right)$. In this case, $\sin \theta=r_{\mathrm{pc}} / R=\left(R / R_{\mathrm{LOFL}}\right)^{1 / 2}$, where $R_{\mathrm{LOFL}}$ is the maximum distance of the points in the last open field line (note $R_{\mathrm{LOFL}} \neq R_{\mathrm{LC}}$, but of the same order).

In Fig. 1, we plot $\Phi_{\max }$ versus $\alpha$ for different $\rho, R$, and $B$ and find that the maximum potential drop $\Phi_{\max }$ varies with inclination angle $\alpha$. For most part in the range 0-90 degrees, $\Phi_{\max }$ is nearly two magnitudes larger than that when $\alpha=0$. The inclination angle of PSR B0943+10 is 12.4 degrees (Lyne \& Manchester 1988). The other parameters with large uncertainty are $\rho$ and $A$. The maximum potential drop $\Phi_{\max }$ is a function of these two. In Fig. 2, we show the potential drop versus $\rho$ and $A$. One can see that the potential drop could be well above $\Phi_{\mathrm{c}}\left(\sim 10^{12} \mathrm{~V}\right)$. 


\section{Conclusion and discussion}

The pulsar PSR B0943+10 could be a low-mass bare quark star of radius $\sim 2.6 \mathrm{~km}$ and mass $\sim 0.02 M_{\odot}$. The polar cap area can fit the observed value of $\sim 10^{3} \mathrm{~m}^{2}$. We have taken into account the effect of inclination angle, which is rarely considered previously. The maximum potential drop for PSR B0943+10 with $\alpha=12.4^{\circ}$ could be $10^{13 \sim 14} \mathrm{~V}$ (Fig. 2). As a consequence, the pulsar's magnetosphere would still be active (i.e., the star would be above the death-line) and thus be radio loud. Our model is only a modification of RS75 model: the only difference is that the central star is a bare quark star rather than a normal neutron star. General features of RS75 keep in our model (e.g., subpulse drifting would still happens). However, there might be two advantages in quark star model: the binding energy problem and the necessary of $\boldsymbol{\Omega} \cdot \mathbf{B}<\mathbf{0}$ in RS75 model do not exist anymore since the binding energy of quarks and electrons here is nearly infinity (Xu \& Qiao 2001).

Could Planck-like emission radiate from the polar caps of bare quark stars? This is a real question in the study of astrophysical quark matter with low temperature but high baryon density, which could be separated into the following two. (i) Could the emissivity of bare strange stars be high enough to produce thermal photon due to high plasma frequency? (ii) Can a bare strange star keep a hot polar cap due to high thermal conductivity? Actually, because of the uncertainties of knowledge about this kind of quark matter, several speculations are proposed. Besides color superconductivity (CSC; e.g., Alford, Bowers \& Rajagopal 2001), Xu (2003b) suggested alternatively that quark matter with low temperature should be in a solid state according to the different manifestations of pulsar-like stars. CSC occurs if quarks are in a condensation in momentum space; quark clusters may form if quarks are in a condensation in position space due to strong interaction, and quark matter could be in a solid state if the temperature is lower than the interaction energy between quark clusters. The plasma frequency derived from fluid quark matter should not be applicable in this solid quark star model. However, the idea that quark matter with low temperature could be in solid state is only a conjecture since no strong constrain is given by experiments or observations. Though QCD is believed to be the theory that would describe quark matter, it is now far from telling us how quark matter behaves. As a result, we also could not know certainly whether quark matter is in a solid or a fluid phase according to today's knowledge. Nevertheless, there could be some hints for solid quark matter, e.g., free precession (e.g., Zhu \& Xu 2006), glitch (e.g., Zhou et al. 2004), and Planckian emission (e.g., Xu 2002; Drake et al. 2002; Burwitz et al. 2003; Xu 2003b).

The observed X-ray spectrum can also be fitted by a power law (Zhang et al. 2005), which could indicate emission from magnetosphere. If only part of X-rays is the thermal emission from the polar cap, the derived cap radius becomes smaller, and our model still works. It needs further observations to determine whether the emission is thermal or non-thermal or has both components. At the same time, thermal emission does not mean blackbody emission. Although the ultra deep observations of RX J1856-3754 (from both Chandra and XMM-Newton, e.g., Burwitz et al. 2003) showed a very high-quality Planck-like spectrum, which may hint a quark surface, blackbody emission is only an assumption for simplicity since we do not know the emissivity exactly. For PSR B0943+10, if its 
thermal emissivity is $\sim 60$ times smaller than that of blackbody, a star of $\sim 10 \mathrm{~km}$ would also be possible, but it is serious problem to obtain theoretically such a low emissivity for neutron star atmospheres with ions and electrons. Could the emissivity of quark star surface be significantly small? If this is the possible, the star's radius would be larger than $\sim 2.6 \mathrm{~km}$.

There are two correction factors for the polar cap area. One is the projection factor. Actually, the observation shows only a projected area (Zhang et al. 2005), but the real polar cap area could be larger since we are not situated at the direction of the magnetic axis. The polar cap area should be divided by a projection factor. It can be estimated as $[\cos (\zeta+\alpha)+\cos (\zeta-\alpha)] / 2 \simeq 0.93$. The modification is smaller than $10 \%$. Hence the conclusions presented would not change. The other factor comes from the shape of the polar cap, because it is not an exact circle when $\alpha \neq 0$. Since $\alpha\left(=12.4^{\circ}\right)$ is small, this effect is also negligible (Qiao et al. 2004).

We use $\theta_{1}$ and $\theta_{2}$ as the boundary angle (see the definitions under eq.[2]). In this way we get the maximum potential drop. The effective potential drop depends on how the charge current flows, which can hardly be derived from first principles. If we use $\alpha$ instead of $\theta_{1}$, as in the conventional cases, the maximum potential drop will be about half the value. However, this does not affect our conclusion significantly because the effective potential drop should be a few $\times 10^{12} \mathrm{~V}$ (which is just above $\Phi_{\mathrm{c}}$, e.g., RS75; Usov \& Melrose 1995). Once the gap potential drop increases to $\gtrsim \Phi_{\mathrm{c}}$, sparks forms, the gap discharges, and the potential decreases. As particles flow out, the potential drop increases again. Thus, if maximum potential is $>\Phi_{\mathrm{c}}$, the RS75-type vacuum gap model works.

The $B \propto(P \dot{P})^{1 / 2}$ approximation is derived by assuming pure magnetic dipole radiation $(\alpha=$ $90^{\circ}$ ), where the effect of $\alpha$ is not included. Actually, there could be two different braking mechanisms for isolated radio pulsars to spin down: the Poynting flux of the magnetodipole radiation and the relativistic particle ejection due to the unipolar generator. The observed braking index (between 1.4 and 2.9, Livingstone et al. 2006, and references therein) could be naturally understood if these two mechanisms are combined (Xu \& Qiao 2001; Contopoulos \& Spitkovsky 2006). These different torques result in spindown-powers of a same order. Spitkovsky (2006) has shown that the pulsar luminosity has a weak dependence on $\alpha: L \propto 1+\sin ^{2} \alpha$. He uses a simple dipole magnetic field configuration which is also assumed in RS75 and in our model. His result could also be applicable for quark star because a quark star may gain a dipole magnetic field by spontaneous magnetization (Xu 2005). The only difference is that a quark star could be of low mass. The polar magnetic field can be express as (note that Spitkovsky (2006) calculated for the magnetic field at the magnetic equator while we do for the polar magnetic field),

$$
B=5.2 \times 10^{19}(P \dot{P})^{1 / 2}\left(\frac{M}{1.4 M_{\odot}}\right)^{1 / 2}\left(\frac{R}{10 \mathrm{~km}}\right)^{-2}\left(1+\sin ^{2} \alpha\right)^{-1 / 2} \quad \mathrm{G} .
$$

The dependence on $\alpha$ is weak: $B$ only differs by a factor of $\sqrt{2}$ at most. Thus the $B \propto(P \dot{P})^{1 / 2}$ approximation is applicable. Meanwhile, the value from equation (3) is quite close to equation (1).

Though Deshpande \& Rankin $(1999,2001)$ has addressed a measured drifting period of sparks on the cap of PSR B0943+10, it is still a complex and controversial problem to detect the real 
drifting rate. The the observed drifting period may possibly not be the real one because of the aliasing effect (e.g., Gil et al. 2003; Esamdin et al. 2005): several different drifting rates can fit well the same observation. Therefore, a relatively faster drifting rate might not be a serious problem in this bare quark star model as well as in the RS75 model.

We are grateful to very helpful communications with an anonymous referee. We also would like to thank Dr. Bing Zhang for his comments and suggestions and to acknowledge various stimulating discussions in the pulsar group of Peking university. This work is supported by National Nature Science Foundation of China (10573002) and by the Key Grant Project of Chinese Ministry of Education (305001).

\section{REFERENCES}

Alcock, C., Farhi, E., \& Olinto, A. 1986, ApJ, 310, 261

Alford, M., Bowers, J., \& Rajagopal, K. 2001, Phys. Rev. D, 63, 074016

Arons, J., \& Scharlemenn, E. T. 1979, ApJ, 231, 854

Bodmer A. R. 1971, Phys. Rev. D, 4, 1601

Burwitz, V., Haberl, F., Neuhäser, R., Predehl, P., Trümper, J., \& Zavlin, V. E. 2003, A\&A, 399, 1109

Cheng, K. S., Ho, C., \& Ruderman, M. 1986, ApJ, 300, 500

Contopoulos, I., \& Spitkovsky, A. 2006, ApJ, 643, 1139

Deshpande, A. A., \& Rankin, J. M. 1999, ApJ, 524, 1008

—. 2001, MNRAS, 322, 438

Drake, J. J. et al. 2002, ApJ, 572, 996

Esamdin, A., Lyne, A. G., Graham-Smith, F., Kramer, M., Manchester, R. N., \& Wu, X. 2005, MNRAS, 356, 59

Gil, J., Melikidze, G. I., \& Geppert, U. 2003, A\&A, 407, 315

Gil, J., Melikidze, G. I., \& Mitra, D. 2002, A\&A, 388, 246

Gil, J., Melikidze, G. I., \& Zhang, B. 2005, preprint (astro-ph/0512653)

Gil, J., Melikidze, G. I., \& Zhang, B. 2006, preprint (astro-ph/0601613)

Itoh, N. 1970, Progress of Theoretical Physics, 44, 291 
Ivanenko, D., \& Kurdgelaidze, D. F. 1969, Lett. Nuovo Cimento, 2, 13

Lai, D., \& Salpeter, E. E. 1997, ApJ, 491, 270

Livingstone, M. A. , Kaspi, V. M., Gotthelf, E. V., Kuiper, L. 2006, priprint (astro-ph/0601530)

Lyne, A. G., \& Manchester, R. N. 1988, MNRAS, 234, 477

Qiao, G. J., Lee, K. J., Wang, H. G., Xu, R. X., \& Han, J. L. 2004, ApJ, 606, L49

Ruderman, M., \& Sutherland, P. G. 1975, ApJ, 196, 51 (RS75)

Spitkovsky, A. 2006, preprint (astro-ph/0603147)

Turolla, R., Zane, S., \& Drake, J. 2004, ApJ, 603, 265

Usov, V. V., \& Melrose, D. B. 1995, Aust. J. Phys., 48, 571

Witten E. 1984, Phys. Rev. D, 30, 272

Xu, R. X. 2002, ApJ, 570, L65

Xu, R. X. 2003a, in: High Energy Processes, Phenomena in Astrophysics, Proceedings of IAU Symposium No. 214, ed. X. D. Li, Z. R. Wang, \& V. Trimble, 191

Xu, R. X. 2003b, ApJ, 596, L59

Xu, R. X. 2005, MNRAS, 356, 359

Xu, R. X., Qiao, G. J. 1998, Chin. Phys. Lett., 15, 934, preprint (astro-ph/9811197)

Xu, R. X., \& Qiao, G. J. 2001, ApJ, 561, L85

Xu, R. X., Qiao, G. J., \& Zhang, B. 1999, ApJ, 522, L109

Xu, R. X., Zhang, B., \& Qiao, G. J. 2001, Astroparticle Physics, 15, 101

Zhang, B., Sanwal, D., \& Pavlov, G. G. 2005, ApJ, 624, L109

Zhou, A. Z., Xu, R. X., Wu, X. J., \& Wang, N. 2004, Astroparticle Physics, 22, 73

Zhu, W. W., \& Xu, R. X. 2006, MNRAS, 365, L16 


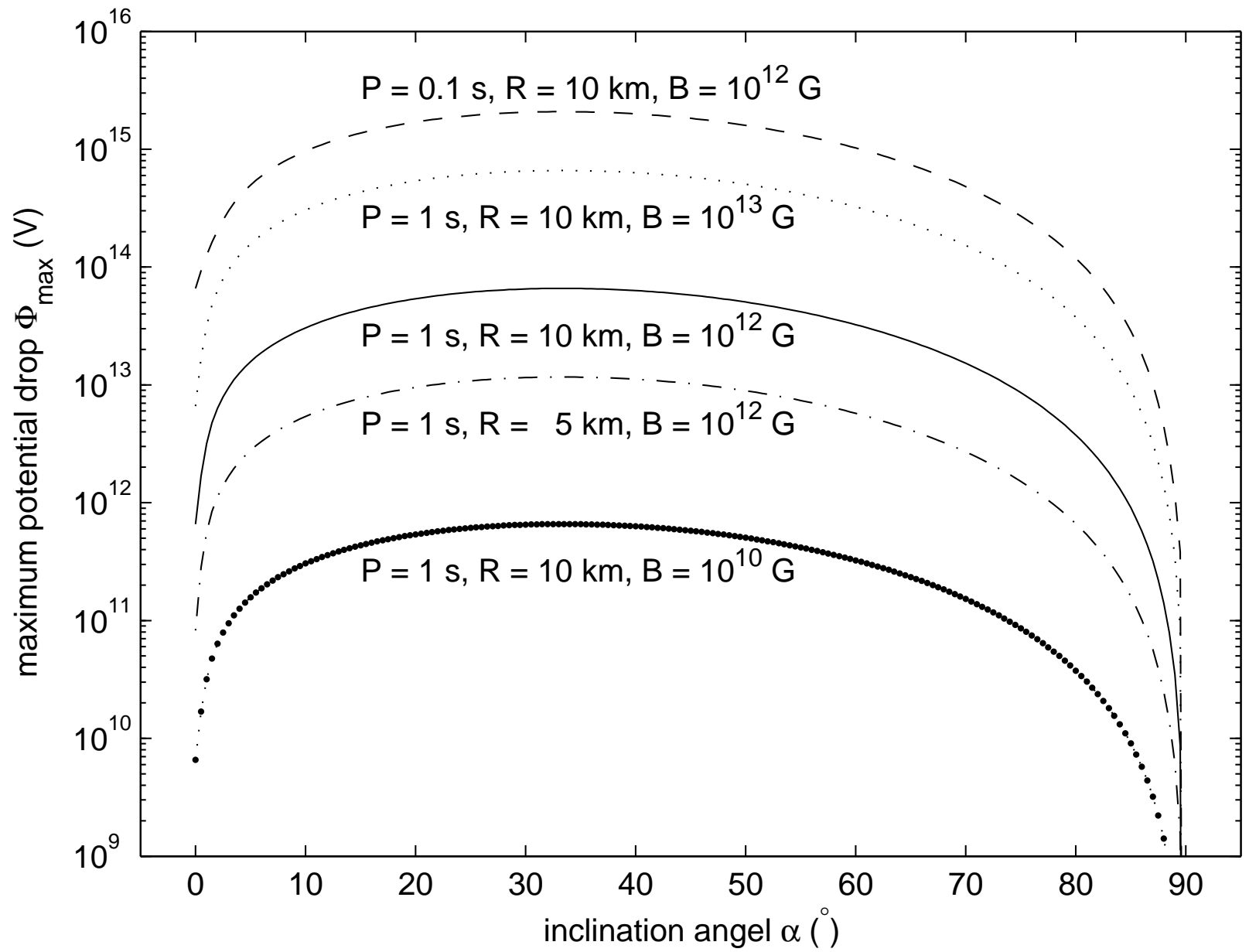

Fig. 1. - Maximum potential drops in the last open field line region for different spin period $(P)$, radius $(R)$, and magnetic field strength $(B)$. 


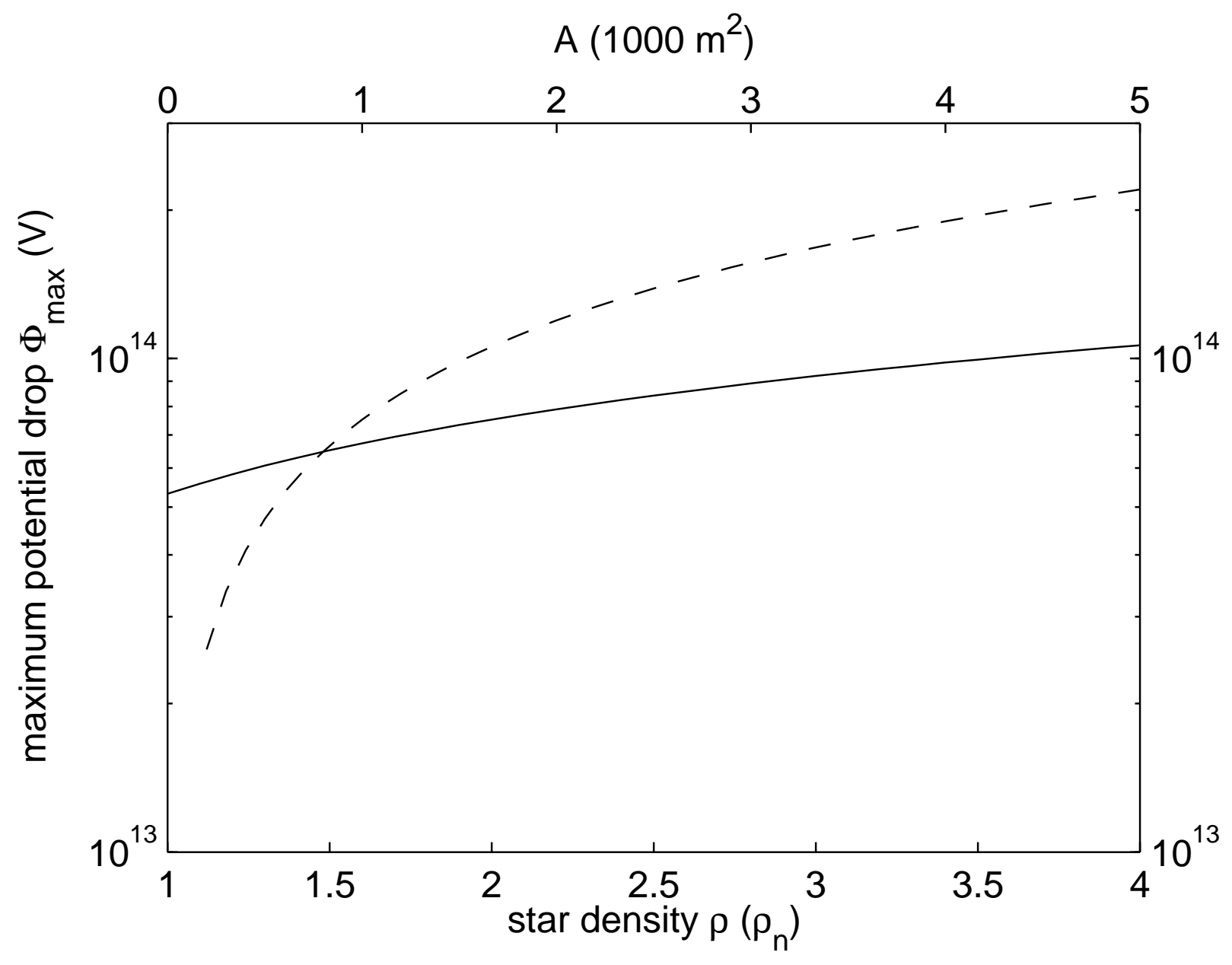

Fig. 2.- Maximum potential drops $\Phi_{\max }$ versus stellar density $\rho$ (solid line) and polar cap area $A$ (dashed line) of PSR B0943+10. We use inclination angle $\alpha=12.4^{\circ}$ here. The area, $A$, is in the range of $0.3 \times 10^{3}-5 \times 10^{3} \mathrm{~m}^{2}$ which is the $1-\sigma$ uncertainty range of the observation from Zhang et al. (2005). The value $A=10^{3} \mathrm{~m}^{2}$ is used for calculating the solid line; $\rho=2 \rho_{\mathrm{n}}$ is fixed for the dashed line. It is evident that $\Phi_{\max }$ is well above $10^{12} \mathrm{~V}\left(\sim \Phi_{\mathrm{c}}\right)$. 